\title{
HIV Risk Perception among HIV Negative or Status-Unknown Men Who Have Sex with Men in China
}

\author{
Wensheng Fan, ${ }^{1}$ Lu Yin, ${ }^{2}$ Han-Zhu Qian, ${ }^{2,3}$ Dongliang Li, ${ }^{4}$ Yiming Shao, ${ }^{5}$ \\ Sten H. Vermund, ${ }^{2,6}$ Yuhua Ruan, ${ }^{5}$ and Zheng Zhang ${ }^{4}$ \\ ${ }^{1}$ Department of Public Health, College of Health and Human Services, Western Kentucky University, Bowling Green, KY 42101, USA \\ ${ }^{2}$ Vanderbilt Institute for Global Health, Vanderbilt University School of Medicine, Nashville, TN 37203, USA \\ ${ }^{3}$ Department of Medicine, Vanderbilt University School of Medicine, Nashville, TN 37203, USA \\ ${ }^{4}$ Chaoyang Center for Disease Control and Prevention, 25 Huaweili, Panjiayuan, Chaoyang District, Beijing 100021, China \\ ${ }^{5}$ State Key Laboratory for Infectious Disease Prevention and Control, National Center for AIDS/STD Control and Prevention, \\ Chinese Center for Disease Control and Prevention, Collaborative Innovation Center for Diagnosis and Treatment of Infectious \\ Diseases, 155 Changbai Road, Changping District, Beijing 102206, China \\ ${ }^{6}$ Department of Pediatrics, Vanderbilt University School of Medicine, Nashville, TN 37203, USA
}

Correspondence should be addressed to Yuhua Ruan; ruanyuhua92@gmail.com and Zheng Zhang; anggie_zhzh@126.com

Received 5 October 2013; Accepted 25 November 2013; Published 30 March 2014

Academic Editor: Muktar Aliyu

Copyright (C) 2014 Wensheng Fan et al. This is an open access article distributed under the Creative Commons Attribution License, which permits unrestricted use, distribution, and reproduction in any medium, provided the original work is properly cited.

Objective. To evaluate HIV risk perception and its associated factors among Chinese MSM. Methods. A cross-sectional study was conducted among MSM with an HIV negative or unknown status in Beijing, China, between 2011 and 2012. A questionnaire interview was conducted and a blood sample was collected for HIV and syphilis testing. Results. Of 887 MSM who reported they were HIV negative or did not know their HIV status before recruitment, only 7.3\% reported a high risk of HIV infection, $28.0 \%$ medium risk, $52.2 \%$ low risk, and $12.5 \%$ no risk. In multivariate logistic regression models using those who reported a medium self-perceived risk as a reference group, self-reported high risk of HIV perception was associated with minority ethnicity (odds ratio [OR]: 2.91; 95\% confidence interval [CI]: 1.03-8.19), self-reported history of sexually transmitted diseases (OR: $2.27 ; 95 \%$ CI: 1.25-4.10), and HIV testing times since the last HIV testing (OR: 0.47; 95\% CI: 0.26-0.84); low self-perceived risk of HIV infection was related to full-time employment (OR: 1.58; 95\% CI: 1.15-2.18) and illicit drug use (OR: 0.28; 95\% CI: 0.10-0.75). Conclusions. The HIV/AIDS epidemic is rapidly rising among Beijing MSM, but more than half MSM did not perceive this risk.

\section{Introduction}

Data from Europe, North America, Latin America, Asia, and Sub-Saharan Africa indicate that human immunodeficiency virus/acquired immunodeficiency syndrome (HIV/AIDS) has been increasing over time, especially among men who have sex with men (MSM) [1-5]. In China, $17.4 \%$ were infected through homosexual contacts in 2011, rising from $11.0 \%$ in 2007 to $7.3 \%$ in 2005 [6]. Three prospective cohort studies which had been separately conducted among MSM in Beijing during 2006-2010 and followed for one year with more than $86.0 \%$ retention reported 2.6, 3.4, and 8.1 per 100 person-years of HIV incidence rates in 2007, 2009, and 2010, respectively [7-9]. Four cross-sectional studies showed that
HIV prevalence rate among MSM in Beijing significantly increased to $6.3 \%$ in 2009 , from $5.8 \%$ in $2006,4.6 \%$ in 2005 , and $0.4 \%$ in 2004 , representing a 15 -times increase within 5 years from 2004 to 2009 [10, 11]. The rapid increase of HIV epidemic among MSM has reminded the Chinese government and researchers that comprehensive biomedical and behavioral interventions and other policies are urgently needed for preventing the spread of HIV in this high risk population.

Age, unmarried status, education, multiple or temporary sexual partners, and inconsistent condom use have been reported to be related to HIV risk among MSM [7, 11, 12]. Since China has adopted an open-door policy since the early 1980s, the Chinese society has become more tolerant 
towards sex including homosexuality. Lack of sex knowledge and safer sex awareness among young people [13] and pursuit of sexual pleasure without condom use among MSM are prevalent, even though various policies, strategies, and interventions for HIV/AIDS prevention and control have been introduced and applied to the MSM population [710]. In addition, unprotected sex often occurs under drug or alcohol use among a proportion of MSM [14-16]. Therefore, risk awareness education is especially important to prevent and control HIV transmission among MSM $[17,18]$.

A qualitative study among MSM in Beijing and Chongqing found that the majority of participants were aware of the high HIV epidemic among MSM, but they did not think themselves at a high risk of HIV infection [19]. We conducted a quantitative assessment of HIV risk perception and its associated factors among Chinese MSM.

\section{Methods}

2.1. Study Design and Population. This study was conducted among MSM in Beijing, China, from January 2010 to July 2011. The study design and study population were described in detail elsewhere [20]. In brief, a local gay volunteer grassroots organization-Chaoyang Chinese AIDS Volunteer Group-recruited MSM participants from the community, sexually transmitted disease (STD) clinics, and voluntary HIV counseling and testing clinics using various approaches, such as website advertisements, outreach to MSM-frequented venues (e.g., MSM clubs, bars, and bathhouses), and peerreferrals. The primary aim of this study was to investigate HPV prevalence using genital and anal swab specimens among HIV-infected and uninfected MSM. This paper aimed to estimate a self-perceived risk of HIV infection among HIV seronegative or status-unknown MSM. Hence, those who have known their positive status are excluded from these analyses $(N=233)$. Written informed consent was obtained before the questionnaire interview and blood collection. This study was reviewed and monitored by the Institutional Review Boards of the National Center for AIDS/STD Control and Prevention (NCAIDS) of the Chinese Center for Disease Control and Prevention (NCAIDS IRB FWA00002958) and Vanderbilt University School of Medicine (VU IRB FWA00005756).

2.2. Data Collection. Questionnaire data of sociodemographic factors, drug and alcohol use, preferred sexual position during anal sex, self-reported sexual orientation, multiple concurrent male sexual partners in the last 12 months, traded sex for money in the past 12 months, forced to have sex with any male partners in the past 12 months, selfreported history of sexually transmitted diseases, ever having sex with female sexual partner(s), history of HIV testing, and self-perception of HIV/AIDS risk were collected by welltrained interviewers.

2.3. Blood Collection and Laboratory Tests. A physical examination was conducted by trained and experienced physicians to collect circumcision status. A blood sample was collected to test for HIV and syphilis infections according to Chinese National Testing Protocols at the Institute of STD/AIDS Prevention and Treatment, Xicheng District Center for Disease Control and Prevention and the Beijing Jingcheng Venereal Hospital, Beijing, China. Each participant was assigned a unique code to link the anonymous questionnaire and blood. HIV infection status was determined by an enzyme immunoassay (Wantai Biological Medicine Company, Beijing, China), and positive samples were confirmed by HIV-uninfected 1/2 Western blot assay (HIV Blot 2.2 WB; Genelabs Diagnostics, Singapore, Singapore). Syphilis serology was determined through rapid plasma reagin (Shanghai Kehua Biotechnology Co., Ltd., Shanghai, China) and confirmed by the Treponema palladium particle assay (Fujirebio Inc., Tokyo, Japan).

2.4. Statistical Analysis. Data for questionnaire responses, physical examinations, and laboratory tests were entered independently by 2 study staff and verified with EpiData software (EpiData 3.1 for Windows; The EpiData Association Odense, Odense, Denmark). Completed databases were then analyzed with Statistical Analysis System (SAS 9.3 for Windows; SAS Institute Inc., Cary, NC, USA) software. Participants were asked a question: "How large do you think the risk of being infected with HIV?" And they could respond with one of four answers: (1) high risk; (2) medium risk; (3) low risk; and (4) no risk. Univariate logistic regression analyses were used to estimate the odds ratios (OR) and 95\% confidence interval (CI) for the association between HIV risk perception and some demographic and behavioral factors, using medium risk as reference: (1) high risk versus medium risk; (2) low risk versus medium risk; and (3) no risk versus medium risk. Multivariable logistic regression was used to determine predictors of HIV risk perception using medium risk as the reference. All variables with $P<0.10$ in univariate analyses were entered into the multivariate logistic model using stepwise selection. Separate logistic regression models were used to further evaluate the associations of HIV/syphilis infection with self-perceived risk of HIV infection without and with adjustment for potential confounders, such as age, ethnic, years of education, and full-time employment.

\section{Results}

A total of 1155 potential participants were recruited into the study and provided informed consent; 251 were excluded due to HIV-seropositive or unconfirmed HIV status; 17 were further excluded due to missing information on HIV risk perception. Therefore, a total of $887 \mathrm{HIV}$-uninfected participants were included in this analysis on HIV risk perception.

Table 1 presents basic characteristics of MSM who participated in this study. The average age at interview was 30.2 years; $94.0 \%$ were Han ethnics; $72.9 \%$ were never married; $52.5 \%$ received college education; $63.9 \%$ were employed full time; $2.8 \%$ ever having used illicit drugs; $24.8 \%$ reported alcohol drinking daily in the past 4 weeks. About twothirds $(65.7 \%)$ reported homosexual orientation and $33.1 \%$ 
TABLE 1: Basic characteristics of men who have sex with men (MSM) in Beijing, China.

\begin{tabular}{lcc}
\hline Characteristics $^{\mathrm{a}}$ & $\begin{array}{c}n \\
(N=887)\end{array}$ & $\%$ \\
\hline Age at interview (year): mean $\pm \mathrm{SD}^{\mathrm{b}}$ (range) & $30.2 \pm 7.9$ & $(18-67)$ \\
$\quad \leq 23$ & 148 & 16.7 \\
$24-29$ & 344 & 38.8 \\
$30-39$ & 278 & 31.3 \\
$\geq 40$ & 87 & 13.2 \\
Ethnicity & & \\
$\quad$ Han & 834 & 94.0 \\
Others & 53 & 6.0
\end{tabular}

Ever married

Never

Ever

Education (year)

Primary school and lower $(\leq 6)$

Middle school (7-9)

Senior high (10-12)

College and higher $(>12)$

Employment status

Full-time/tenured

Part-time/temporary

Retired/unemployed

Student

Others

Resident in Beijing

No

Yes

Illicit drug use

Never

Ever

Alcohol drinking in the past 4 weeks

Never

Rarely

2-6 times per week

Daily

Sexual orientation

Homosexual

Heterosexual

Bisexual

Preferred sexual position during anal sex

$\begin{array}{lcc}\text { Exclusively receptive } & 103 & 11.6 \\ \text { Mainly receptive } & 155 & 17.5 \\ \text { Exclusively insertive } & 181 & 20.4 \\ \text { Mainly insertive } & 224 & 25.2 \\ \text { Dual } & 193 & 21.8 \\ \text { No anal sex } & 31 & 3.5\end{array}$

TABLE 1: Continued.

\begin{tabular}{|c|c|c|}
\hline Characteristics $^{\mathrm{a}}$ & $\begin{array}{c}n \\
(N=887)\end{array}$ & $\%$ \\
\hline \multicolumn{3}{|c|}{ Self-perceived risk of HIV infection } \\
\hline High & 65 & 7.3 \\
\hline Medium & 248 & 28.0 \\
\hline Low & 463 & 52.2 \\
\hline No & 111 & 12.5 \\
\hline \multicolumn{3}{|c|}{$\begin{array}{l}\text { HIV testing times since last HIV testing } \\
\text { (month) }\end{array}$} \\
\hline Never & 30 & 3.4 \\
\hline$<3$ & 282 & 31.8 \\
\hline $3-5$ & 326 & 36.8 \\
\hline $6-11$ & 132 & 14.9 \\
\hline$\geq 12$ & 117 & 13.2 \\
\hline \multicolumn{3}{|l|}{ HIV seropositive } \\
\hline No & 840 & 94.7 \\
\hline Yes & 47 & 5.3 \\
\hline \multicolumn{3}{|c|}{ Syphilis seropositive } \\
\hline No & 739 & 83.3 \\
\hline Yes & 148 & 16.7 \\
\hline
\end{tabular}

reported bisexual orientation. As for the role of anal sex, $29.1 \%$ preferred receptive, $45.6 \%$ preferred insertive, and $21.8 \%$ reported dual. Among this study population, only $3.4 \%$ had never had HIV testing and $83.4 \%$ reported having HIV testing in the past 12 months. Of 887 participants, 5.3\% were confirmed as HIV positive and $16.7 \%$ as syphilis positive.

Only $7.3 \%$ perceived themselves at high risk of HIV infection; $28.0 \%$ perceived themselves to be at medium risk; $52.2 \%$ at low risk; and $12.5 \%$ at no risk. In unadjusted analyses, the following factors were associated with high risk perception compared with medium risk perception: younger age (OR, $0.96 ; 95 \% \mathrm{CI}, 0.92-1.00 ; P=0.07)$ and minority ethnicity (OR, 2.87; 95\% CI, 1.05-7.87; $P=0.04$ ), history of sexually transmitted diseases (OR, 2.17; 95\% CI, 1.22-3.88; $P=0.01)$, and $\geq 3$ months since last HIV testing (OR, 0.48; 95\% CI, 0.27-0.84; $P=0.01$ ). Factors associated with low risk perception were higher education level (OR, 1.37; 95\% CI, 1.01-1.87; $P=0.05$ ), full-time employment (OR, 1.64; $95 \%$ CI, 1.19-2.25; $P<0.01$ ), and no history of illicit drug use (OR, $0.26 ; 95 \% \mathrm{CI}, 0.10-0.69 ; P=0.01$ ). Factors associated with no self-perceived risk were lower education level (OR, 0.58; $95 \%$ CI, $0.37-0.92 ; P=0.02$ ), bisexual orientation (OR, 1.79; $95 \%$ CI, 1.11-2.88; $P=0.02)$, and ever had sex with female sexual partner(s) (OR, 2.02; 95\% CI, 1.28-3.17; $P<0.01)$ (Table 2).

The factors significant in the univariate analyses were included in multivariate analyses (Table 3). Compared with those with medium risk perception, those with high risk perception tended to be minority ethnicity (OR, 2.91; 95\% CI, 


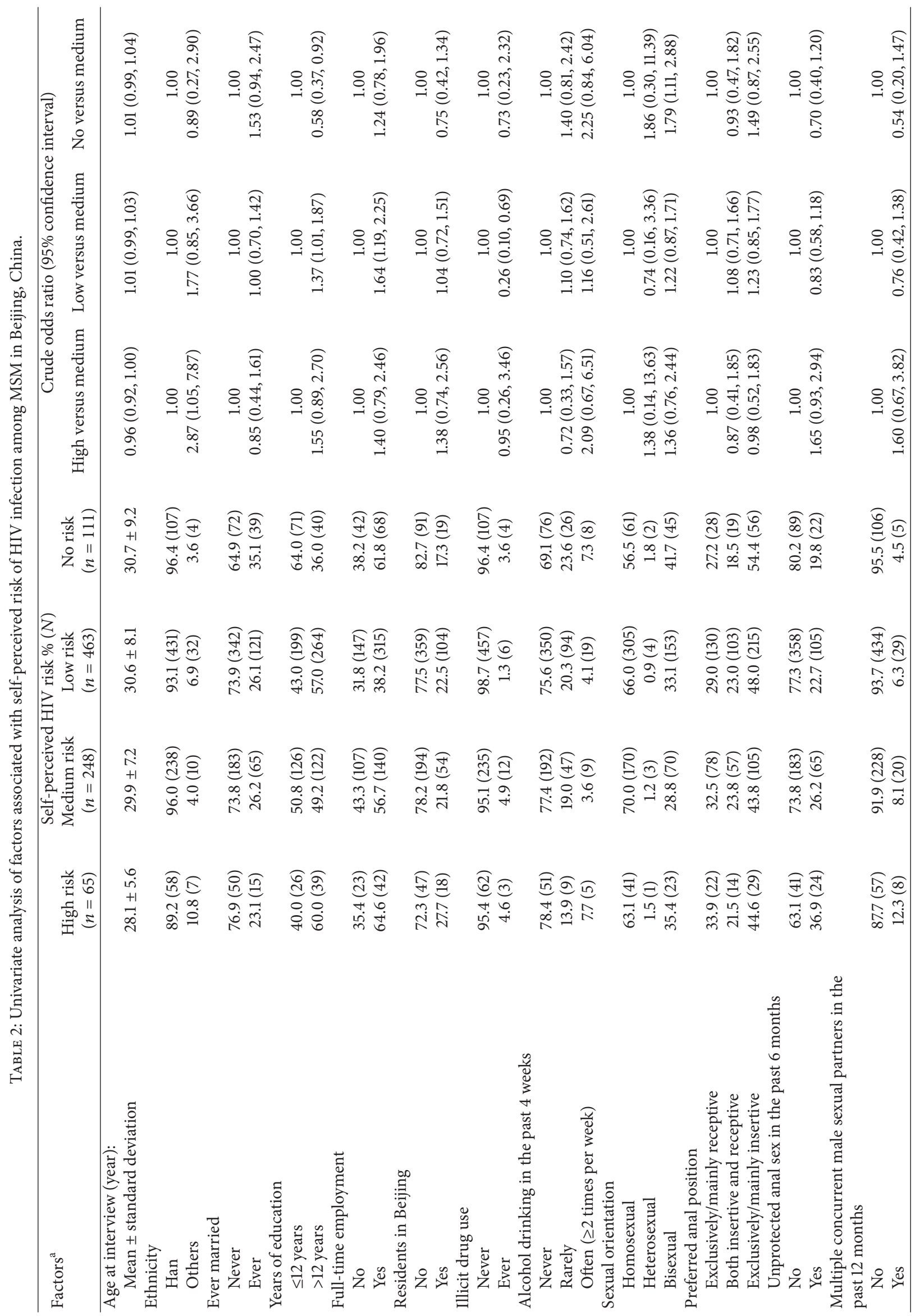




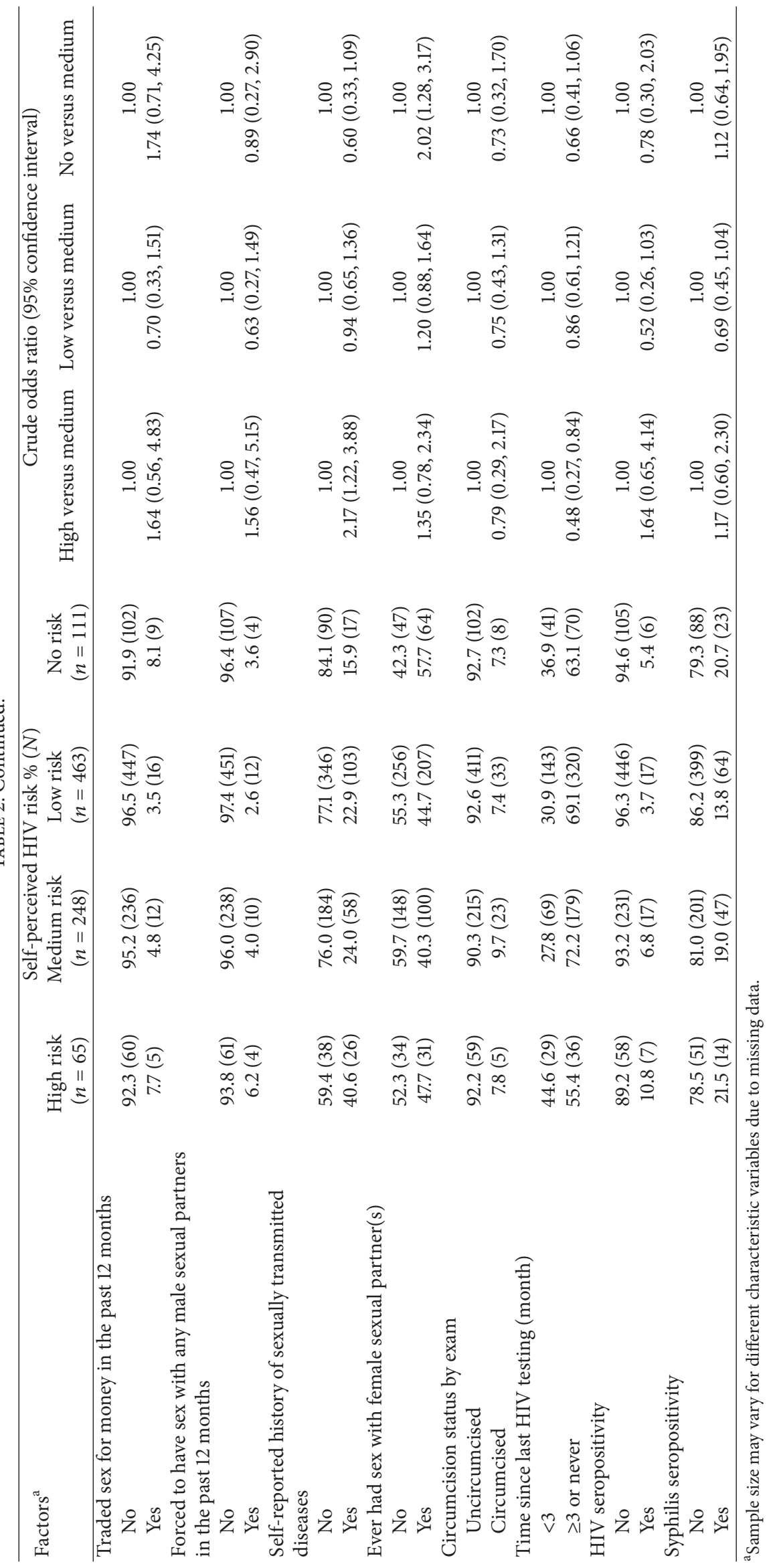


TABLE 3: Multivariate analysis of factors associated with self-perceived risk of HIV infection among MSM in Beijing, China.

\begin{tabular}{|c|c|c|c|}
\hline \multirow{2}{*}{ Factors } & \multicolumn{3}{|c|}{ Adjusted OR (95\% CI) } \\
\hline & High versus medium & Low versus medium & No versus medium \\
\hline \multicolumn{4}{|l|}{ Ethnicity } \\
\hline Others versus Han & $2.91(1.03,8.19)$ & - & - \\
\hline \multicolumn{4}{|l|}{ Full-time employment } \\
\hline Yes versus no & - & $1.58(1.15,2.18)$ & - \\
\hline \multicolumn{4}{|l|}{ Illicit drug use } \\
\hline Ever versus never & - & $0.28(0.10,0.75)$ & - \\
\hline \multicolumn{4}{|c|}{ History of sexually transmitted diseases } \\
\hline Yes versus no & $2.27(1.25,4.10)$ & - & - \\
\hline \multicolumn{4}{|c|}{ Ever had sex with female sexual partner(s) } \\
\hline Yes versus no & - & - & $1.93(1.22,3.05)$ \\
\hline \multicolumn{4}{|c|}{ HIV testing times since last HIV testing (months) } \\
\hline$\geq 3$ or never versus $<3$ & $0.47(0.26,0.84)$ & - & - \\
\hline
\end{tabular}

Note: OR: odds ratio; CI: confidence interval.

TABLE 4: Association of HIV risk perception with HIV and syphilis infections among MSM in Beijing, China.

\begin{tabular}{lccc}
\hline Self-perceived risk of HIV infection & Unadjusted OR $(95 \%$ CI $)$ & $P$ value & Adjusted OR ${ }^{*}(95 \%$ CI $)$ \\
\hline HIV seropositivity & Ref. & & Ref. \\
$\quad$ High risk & $0.61(0.24,1.54)$ & 0.29 & $0.60(0.23,1.54)$ \\
$\quad$ Medium risk & $0.32(0.13,0.79)$ & 0.01 & $0.35(0.14,0.90)$ \\
$\quad$ Low risk & $0.47(0.15,1.48)$ & 0.20 & $0.46(0.15,1.49)$ \\
$\quad$ No risk & & 0.07 & 0.03 \\
$P_{\text {trend }}$ & Ref. & & 0.20 \\
Syphilis seropositivity & $0.85(0.44,1.67)$ & 0.16 \\
$\quad$ High risk & $0.58(0.31,1.12)$ & 0.64 & $0.77(0.39,1.53)$ \\
$\quad$ Medium risk & $0.95(0.45,2.01)$ & 0.10 & $0.53(0.27,1.02)$ \\
$\quad$ Low risk & & 0.90 & $0.78(0.36,1.68)$ \\
$\quad$ No risk & & 0.11 & 0.46 \\
$P_{\text {trend }}$ & & & 0.06 \\
\hline
\end{tabular}

Note: OR: odds ratio; CI: confidence interval.

* Adjusted for age, ethnic, years of education, and full-time employment.

1.03-8.19; $P=0.04)$, have a history of sexually transmitted diseases (OR, 2.27; 95\% CI, 1.25-4.10; $P=0.01$ ), and have more than 3 months since last HIV testing (OR, 0.47; 95\% CI, $0.26-0.84 ; P=0.01)$. Those with low risk perception tended to be a full-time employee (OR, 1.58; 95\% CI, 1.15-2.18; $P=0.01)$ and have a history of using illicit drugs (OR, 0.28; 95\% CI, 0.10-0.75; $P=0.01$ ). Those with no perceived HIV risk were more likely to have female sexual partners (OR, 1.93; 95\% CI, 1.22-3.05; $P=0.01$ ).

Table 4 presents the association between HIV risk perception and HIV and syphilis infections. A U-shape association between HIV seropositivity and self-perceived HIV risk was found, and a lower prevalence of HIV infection was observed among those perceiving themselves at low risk than that among those reporting high HIV risk (3.7\% versus $10.8 \%$; OR, 0.35 ; 95\% CI, 0.14-0.90; $P=0.03$ ) after adjusting for age, ethnicity, years of education, and full-time employment. There is a marginally statistically significant association between syphilis seropositivity and self-perceived risk of HIV infection (low risk versus high risk: $13.8 \%$ versus $21.5 \%$; OR, 0.53; 95\% CI, 0.27-1.02; $P=0.06)$.

\section{Discussions}

Our study found that a very small proportion of MSM in Beijing had high risk perception and about two-thirds perceived themselves at low or no risk. Several previous studies in the USA [18, 21-23] and Netherlands [17] also reported that over half of MSM perceived that they had low or no chance of contracting HIV. However, HIV has been endemic among MSM in Beijing [10, 11, 24]. Our study showed that men with high risk perception did have a higher HIV prevalence $(10.8 \%)$ than those reporting medium or low risk (6.8\% and $3.7 \%$, resp.). A marginally significant U-shape trend also was observed for syphilis seropositivity. Such associations between HIV/syphilis infection and selfperceived risk of HIV infection reminded us that it is urgent to improve health education/interventions and increase their high risk of HIV infection among this population.

Behavioral change theories suggest that self-perception plays an important role in health behavior [25]. Among respondents in our survey, 3.4\% had never been tested for HIV and over two- thirds had a test within the past 6 months. 
There are various reasons for not taking a test among Chinese MSM, such as stigma and fear about learning their HIV status [26-28]. However, the prevalence of HIV testing was high in our study, and the coverage of HIV testing among MSM in Beijing has been expanded in the past two years [29]. Noninfected MSM tend not to take a test because they have low risk perception $[23,26,28]$. Hence, it is suggested that MSM or other high risk populations take HIV testing regularly, especially for those frequently engaging in high risk behaviors [30]. Those perceiving themselves as having a high risk of contracting HIV were more likely to seek HIV testing in the past 3 months, compared to those with perceived medium-, low-, or no risk. Providing routine HIV testing in medical care settings may increase HIV risk perception among MSM [22].

Studies have shown that those engaging in risk behaviors were likely to consider themselves at high risk [26]. In our study, it is interesting to find that a higher proportion of those perceiving themselves at high risk of HIV infection reported engaging in high risk behaviors, for example, $36.9 \%$ of those reporting unprotected anal sex and $12.3 \%$ of those reporting multiple concurrent male sexual partners. However, a moderate number of MSM still believed their chance of contracting HIV was low despite their involvement in risky sex in current and previous studies $[18,21,22]$. Though HIV testing has increased among MSM in Beijing, unprotected anal sex is still common $[7,29]$. It is needed to increase risk perception among Chinese MSM.

Several previous studies found that MSM may be even less likely to be tested for STDs than HIV [31-33]. We did not test for STDs besides syphilis, but 33\% of our study participants reported a history of STDs. Participants with high risk perception had an odds of $>2$ times of reporting a history of STDs than those in other risk perception groups. Hence, the promotion of STD testing can also be used as a complementary strategy to enhance risk perception among MSM.

Sex with both men and women is common among Chinese MSM. Nearly $60 \%$ of participants with no perceived risk of HIV infection reported ever had sex with female sexual partners, higher than those having a medium level of risk perception (40\%). The potential for transmitting HIV from bisexual men to either men or women results largely from less condom use and sex with other MSM and women [34, 35]. Risk perception education should emphasize safe sex with both male and female sex partners.

Over half of our study sample received college education and were employed full time. Overall, there was no statistically significant relationship between education or employment with self-perceived risk, but a number of participants with high education and full-time employment thought that they were unlikely to acquire HIV. The risk awareness education program should consider that (1) MSM with lower educational attainment needs the basic information about the growing HIV/AIDS epidemic among MSM in China and the mechanisms of HIV transmission; (2) those with a higher level of education need more in-depth information about the importance of knowing HIV status through HIV testing and information to dispel incorrect information about HIV risk, for example, (1) AIDS is an irrelevant disease; (2) some partners are less risky than others; and (3) cleaning after sex is one way to prevent from HIV transmission [19].

There are some limitations in our study. First, all questionnaire data were based on self-reporting; study participants may have provided responses based more on social desirability to please the interviewers than actual experiences, especially experiences involving sensitive questions. Therefore, it is unknown whether participants under-reported or over-reported their involvement in risky behaviors. Second, the potential factors for HIV risk perception may not be accurately measured using a single question. Third, the MSM who participated in this study are volunteers recruited using nonrandomized sampling. The study sample may not represent the entire MSM community in Beijing or in China. Finally, the potential reasons of low HIV risk perception were not investigated; qualitative assessment may explore these reasons.

\section{Conclusions}

In summary, this study found that few MSM have high selfperceived HIV risk, even with HIV at an epidemic level among MSM in Beijing, China. HIV prevention programs should emphasize increasing HIV risk awareness among Chinese MSM, particularly those who are unemployed and uneducated.

\section{Conflict of Interests}

The authors declare that there is no conflict of interests regarding the publication of this paper.

\section{Acknowledgments}

This study was sponsored by the National Natural Science Foundation of China (81273188), the Vanderbilt Clinical and Translational Research Scholars (VCTRS) Program (UL1 TR000445 and KL2TR000446) and Grants from U.S. NIH (R01AI09462) and the Ministry of Science and Technology of China (2012ZX10004-904 and 2012ZX10001-002) and Chinese State Key Laboratory for Infectious Disease Development Grant (2012SKLID103).

\section{References}

[1] European Centre for Disease Prevention and Control, WHO Regional Office for Europe, HIV/AIDS Surveillance in Europe 2009, European Centre for Disease Prevention and Control, Stockholm, Sweden, 2010.

[2] National Center for HIV/AIDS, Viral Hepatitis, STD \& TB Prevention, Epidemic of HIVInfection through 2009, Washington, DC, USA, 2010, http://www.cdc.gov/hiv/pdf/risk_ HIV_among_AA_Gay_other.pdf.

[3] F. I. Bastos, C. Cáceres, J. Galvão, M. A. Veras, and E. A. Castilho, "AIDS in Latin America: assessing the current status of the epidemic and the ongoing response," International Journal of Epidemiology, vol. 37, no. 4, pp. 729-737, 2008. 
[4] A. D. Smith, P. Tapsoba, N. Peshu, E. J. Sanders, and H. W. Jaffe, "Men who have sex with men and HIV/AIDS in sub-Saharan Africa," The Lancet, vol. 374, no. 9687, pp. 416-422, 2009.

[5] F. van Griensven and J. W. de Lind van Wijngaarden, "A review of the epidemiology of HIV infection and prevention responses among MSM in Asia," AIDS, vol. 24, no. 3, pp. S30-S40, 2010.

[6] Ministry of Health in People's Republic of China, Joint United Nations Programme on HIV/AIDS, World Health Organization, 2011 Estimates for the HIV/AIDS Epidemic in China, Beijing, China, 2011.

[7] Y. Ruan, Y. Jia, X. Zhang et al., "Incidence of HIV-1, syphilis, hepatitis $\mathrm{B}$, and hepatitis $\mathrm{c}$ virus infections and predictors associated with retention in a 12-month follow-up study among men who have sex with men in Beijing, China," Journal of Acquired Immune Deficiency Syndromes, vol. 52, no. 5, pp. 604610, 2009.

[8] S.-M. Li, Z.-H. Zhou, S.-L. Jiang et al., "Incidence and risk factors of HIV and syphilis seroconversion among men who have sex with men in Beijing," Zhonghua Yu Fang Yi Xue Za Zhi, vol. 45, no. 2, pp. 118-122, 2011 (Chinese).

[9] D. Li, S. Li, Y. Liu et al., "HIV incidence among men who have sex with men in Beijing: a prospective cohort study," BMJ Open, vol. 2, no. 6, Article ID e001829, 2012.

[10] X. Ma, Q. Zhang, X. He et al., “Trends in prevalence of HIV, syphilis, hepatitis $\mathrm{C}$, hepatitis $\mathrm{B}$, and sexual risk behavior among men who have sex with men: results of 3 consecutive respondent-driven sampling surveys in Beijing, 2004 through 2006," Journal of Acquired Immune Deficiency Syndromes, vol. 45, no. 5, pp. 581-587, 2007.

[11] D. L. Li, Y. J. Gao, M. R. Yu et al., "Study on the incidence of HIV and associated risk factors through a prospective cohort among men who have sex with men in Beijing, China," Zhonghua Liu Xing Bing Xue Za Zhi, vol. 33, pp. 663-666, 2012 (Chinese).

[12] Y. J. Liu, S. L. Jiang, Y. Hu, L. Song, M. Yu, and S. M. Li, "Characteristics of sexual behaviors and infection status of AIDS and other sexually transmitted diseases among men who have sex with men in 2009 in Beijing," Zhonghua Yu Fang Yi Xue Za Zhi, vol. 45, pp. 971-974, 2011 (Chinese).

[13] J. Yu, "Teenage sexual attitudes and behaviour in China: a literature review," Health and Social Care in the Community, vol. 20, no. 6, pp. 561-582, 2012.

[14] G. B. Gerbi, T. Habtemariam, B. Tameru, D. Nganwa, and V. Robnett, "The correlation between alcohol consumption and risky sexual behaviours among people living with HIV/AIDS," Journal of Substance Use, vol. 14, no. 2, pp. 90-100, 2009.

[15] P. A. Shuper, N. Joharchi, H. Irving, and J. Rehm, "Alcohol as a correlate of unprotected sexual behavior among people living with HIV/AIDS: review and meta-analysis," AIDS and Behavior, vol. 13, no. 6, pp. 1021-1036, 2009.

[16] G. B. Gerbi, T. Habtemariam, B. Tameru, D. Nganwa, and V. Robnett, "A comparative study of substance use before and after establishing HIV infection status among people living with HIV/AIDS," Journal of Substance Use, vol. 16, no. 6, pp. 464-475, 2011.

[17] E. M. van der Snoek, J. B. F. de Wit, H. M. Gotz, P. G. H. Mulder, M. H. A. Neumann, and W. I. van der Meijden, "Incidence of sexually transmitted diseases and HIV infection in men who have sex with men related to knowledge, perceived susceptibility, and perceived severity of sexually transmitted diseases and HIV infection: Dutch MSM-cohort study," Sexually Transmitted Diseases, vol. 33, no. 3, pp. 193-198, 2006.
[18] H. Klein and D. L. Tilley, "Perceptions of HIV risk among internet-using, HIV-negative barebacking men," American Journal of Men's Health, vol. 6, pp. 280-293, 2012.

[19] W. Ma, X. Ding, H. Lu et al., "HIV risk perception among men who have sex with men in two municipalities of Chinaimplications for education and intervention," AIDS Care, vol. 25, pp. 385-389, 2013.

[20] Y. Hu, H. Z. Qian, J. Sun et al., "Anal human papillomavirus infection among HIV-infected and uninfected men who have sex with men in Beijing, China," Journal of Acquired Immune Deficiency Syndromes, vol. 64, no. 1, pp. 103-114, 2013.

[21] D. A. MacKellar, L. A. Valleroy, G. M. Secura et al., "Unrecognized HIV infection, risk behaviors, and perceptions of risk among young men who have sex with men: opportunities for advancing HIV prevention in the third decade of HIV/AIDS," Journal of Acquired Immune Deficiency Syndromes, vol. 38, no. 5, pp. 603-614, 2005.

[22] J. A. Lapidus, J. Bertolli, K. McGowan, and P. Sullivan, "HIVrelated risk behaviors, perceptions of risk, HIV testing, and exposure to prevention messages and methods among urban American Indians and Alaska Natives," AIDS Education and Prevention, vol. 18, no. 6, pp. 546-559, 2006.

[23] D. A. MacKellar, L. A. Valleroy, G. M. Secura et al., "Perceptions of lifetime risk and actual risk for acquiring HIV among young men who have sex with men," AIDS and Behavior, vol. 11, no. 2, pp. 263-270, 2007.

[24] H. Lu, Y. Han, X. He et al., "Alcohol use and HIV risk taking among Chinese MSM in Beijing," Drug and Alcohol Dependence, vol. 133, no. 2, pp. 317-323, 2013.

[25] J. O. Prochaska and W. F. Velicer, "The transtheoretical model of health behavior change," American Journal of Health Promotion, vol. 12, no. 1, pp. 38-48, 1997.

[26] Centers for Disease Control and Prevention (CDC), "Unrecognized HIV infection, risk behaviors, and perceptions of risk among young black men who have sex with men-six U.S. cities, 1994-1998," MMWR. Morbidity and Mortality Weekly Report, vol. 51, no. 33, pp. 733-736, 2002.

[27] A. B. Hutchinson, G. Corbie-Smith, S. B. Thomas, S. Mohanan, and C. Del Rio, "Understanding the patient's perspective on rapid and routine HIV testing in an inner-city urgent care center," AIDS Education and Prevention, vol. 16, no. 2, pp. 101114,2004

[28] J. A. Nel, H. Yi, T. G. Sandfort, and E. Rich, "HIV-untested men who have sex with men in South Africa: the perception of not being at risk and fear of being tested," AIDS and Behavior, vol. 17, pp. S51-S59, 2013.

[29] X. Li, H. Lu, H. F. Raymond et al., "Untested and undiagnosed: barriers to HIV testing among men who have sex with men, Beijing, China," Sexually Transmitted Infections, vol. 88, no. 3, pp. 187-193, 2012.

[30] K. H. Reilly, A. Neaigus, S. M. Jenness, H. Hagan, T. Wendel, and C. Gelpi-Acosta, "High HIV prevalence among low-income, black women in New York City with self-reported HIV negative and unknown status," Journal of Women's Health, vol. 22, no. 9, pp. 745-754, 2013.

[31] E. Tai, T. Sanchez, A. Lansky, K. Mahle, J. Heffelfinger, and K. Workowski, "Self-reported syphilis and gonorrhoea testing among men who have sex with men: national HIV behavioural surveillance system, 2003-5," Sexually Transmitted Infections, vol. 84, no. 6, pp. 478-482, 2008.

[32] C. V. Johnson, M. J. Mimiaga, S. L. Reisner et al., "Health care access and sexually transmitted infection screening frequency 
among at-risk Massachusetts men who have sex with men," American journal of public health, vol. 99, pp. S187-192, 2009.

[33] M. J. Mimiaga, S. L. Reisner, S. Bland et al., "Health system and personal barriers resulting in decreased utilization of HIV and STD testing services among at-risk black men who have sex with men in Massachusetts," AIDS Patient Care and STDs, vol. 23, no. 10, pp. 825-835, 2009.

[34] P. M. Gorbach, R. Murphy, R. E. Weiss, C. Hucks-Ortiz, and S. Shoptaw, "Bridging sexual boundaries: men who have sex with men and women in a street-based sample in Los Angeles," Journal of Urban Health, vol. 86, no. 1, pp. S63-S76, 2009.

[35] M. Sheehy, W. Tun, L. Vu, S. Adebajo, O. Obianwu, and A. Karlyn, "High levels of bisexual behavior and factors associated with bisexual behavior among men having sex with men (MSM) in Nigeria," AIDS Care, vol. 26, no. 1, pp. 116-122, 2013. 


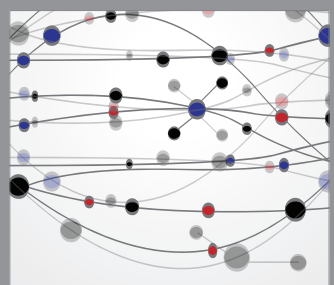

The Scientific World Journal
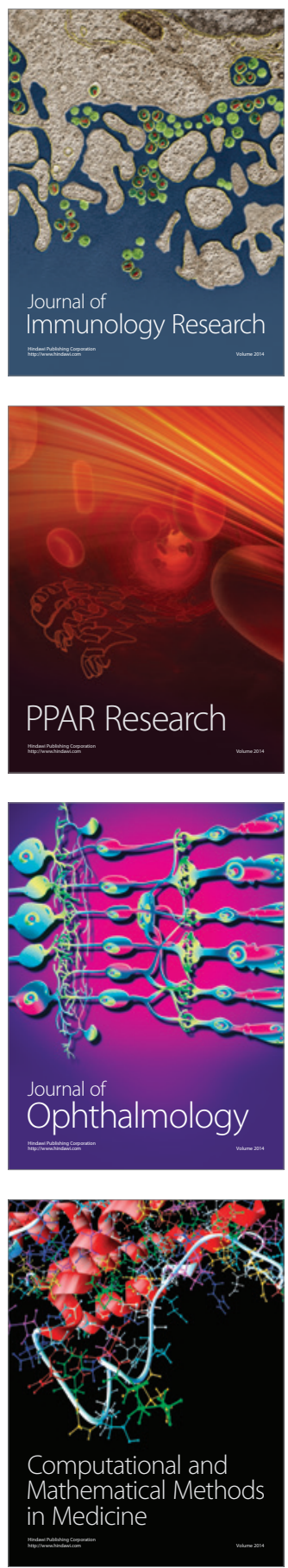

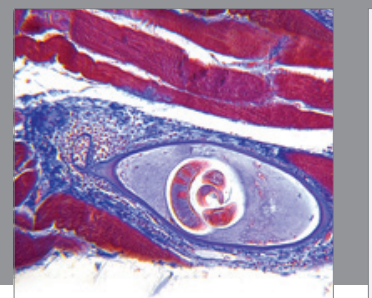

Gastroenterology

Research and Practice
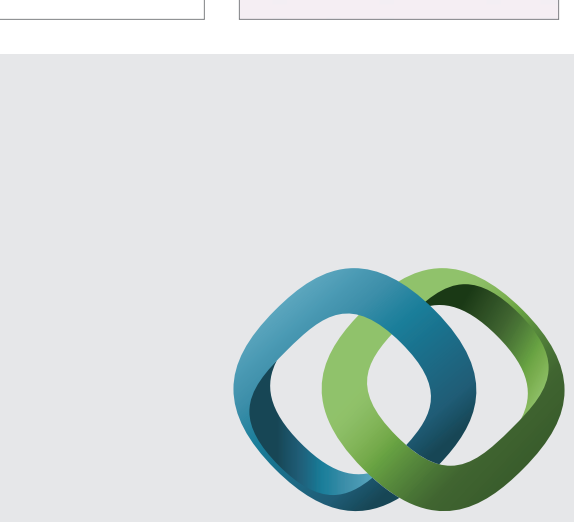

\section{Hindawi}

Submit your manuscripts at

http://www.hindawi.com
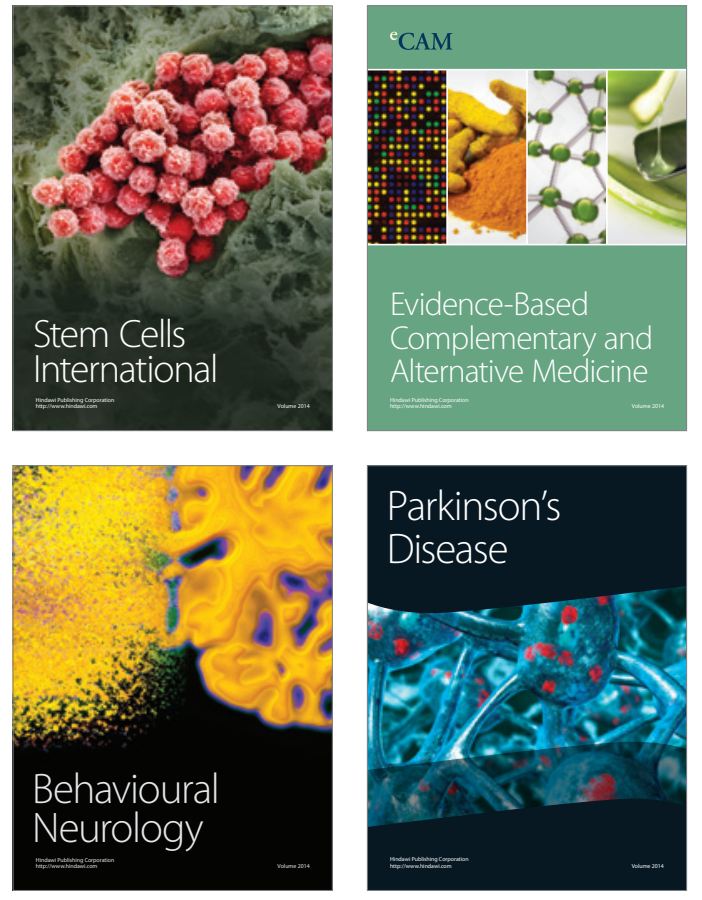
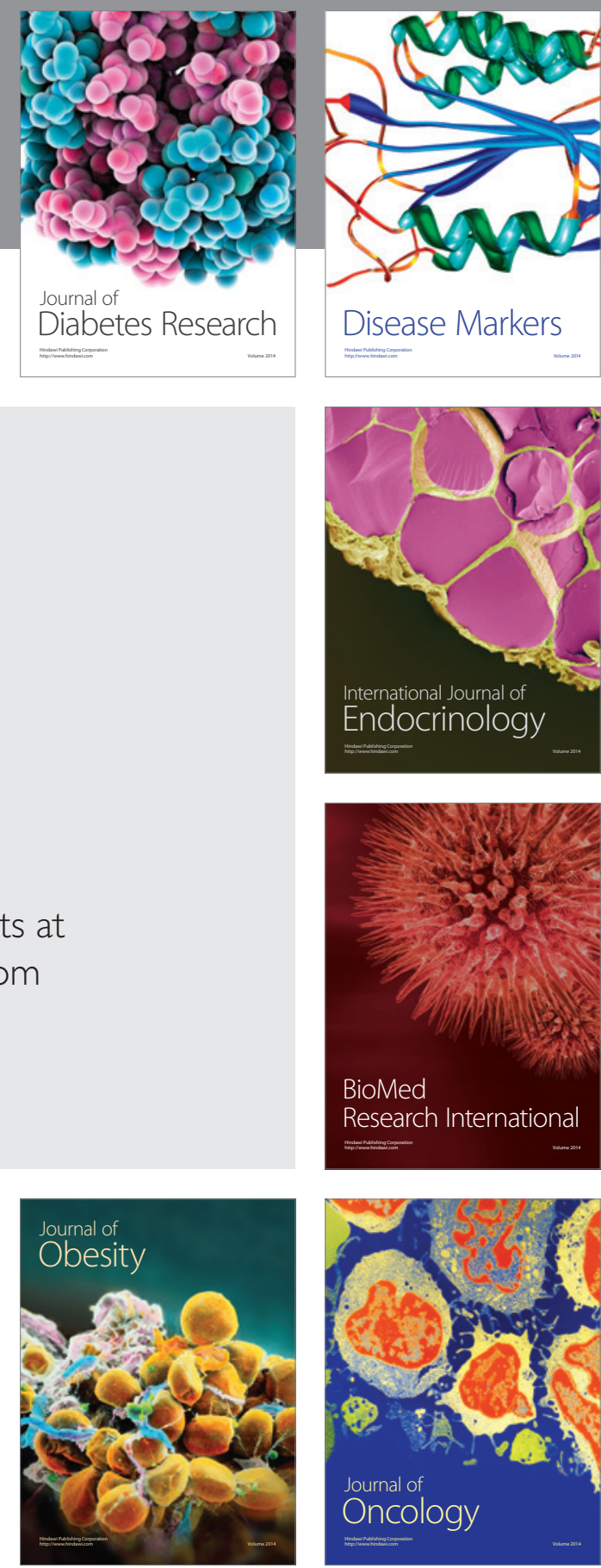

Disease Markers
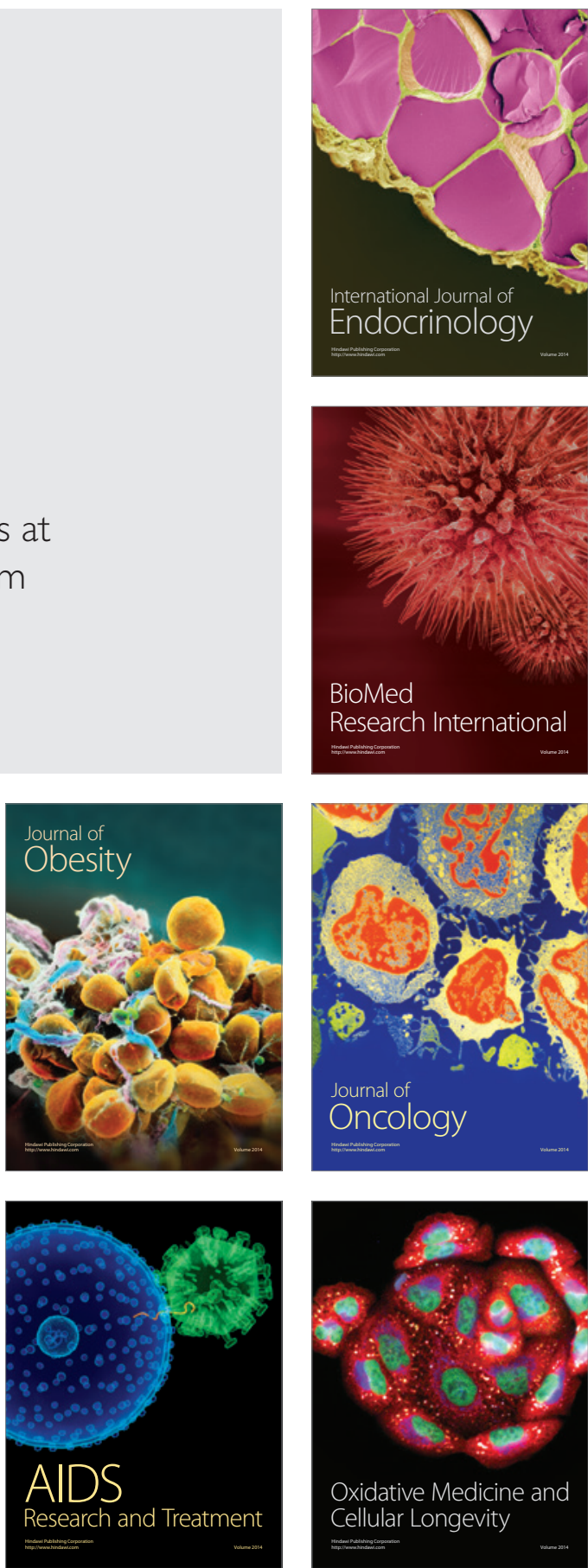